Quim. Nova, Vol. 31, No. 5, 1020-1026, 2008

\title{
FITODISPONIBILIDAD DE Cd EN SUELO DE DIFERENTE pH TRATADO CON LODO ALBAÑAL ESTIMADA POR MÉTODO ISOTÓPICO Y EXTRACCIÓN QUÍMICA
}

\author{
Felipe Carlos Alvarez Villanueva*, Antonio Enedi Boaretto, Cassio Hamilton Abreu Junior, Takashi Muraoka y Anderson \\ Ricardo Trevizam \\ Divisão de Produtividade Agroindustrial e Alimentos, Centro de Energia Nuclear na Agricultura, Universidade de São Paulo, \\ CP 96, 13400-970 Piracicaba - SP, Brasil \\ Virgilio Franco do Nascimento Filho \\ Divisão de Desenvolvimento de Métodos e Técnicas Analíticas e Nucleares, Centro de Energia Nuclear na Agricultura, \\ Universidade de São Paulo, CP 96, 13400-970 Piracicaba - SP, Brasil
}

Recebido em 5/3/07; aceito em 26/10/07; publicado na web em 2/7/08

\begin{abstract}
Cd PHYTOAVAILABILITY IN SEWAGE SLUDGE-AMENDED SOIL OF DIFFERENT pH ESTIMATED BY AN ISOTOPIC METHOD AND CHEMICAL EXTRACTION. The Cd phytoavailability in sewage sludge-amended soils of different pHs using the ${ }^{109} \mathrm{Cd} L$-value isotopic method and $\mathrm{Cd}$ extracted by DTPA has been determined. Maize plants (Zea mais L.) were grown under greenhouse conditions in a xanthic ferralsol at different $\mathrm{pHs}$ amended with five sewage sludge (SS) rates, and labeled with $74 \mathrm{kBq}$ $\mathrm{kg}^{-1}$ of ${ }^{109} \mathrm{Cd}$. The SS rates altered the properties of the soil chemicals and these influenced the isotopic parameter $(L$-value) and percent of Cd uptake by plants from soil (\%Cdpdfs) and SS (\%CdpdfSS). $L$-values and Cd extracted by DTPA correlate significantly with SS rates and Cd uptake by plants and are efficient for predicting the Cd phytoavailability in the sewage sludge-amended soil.
\end{abstract}

Keywords: cadmium; $L$-value; sewage sludge.

\section{INTRODUCCIÓN}

Entre los elementos no esenciales para el desarrollo de sistemas vivos, el cadmio $(\mathrm{Cd})$, por su potencial tóxico, ${ }^{1}$ es el de mayor preocupación en relación al sistema suelo-planta y la entrada en la cadena alimentar. Su presencia en los suelos tiene origen geoquímica y antropogénico debido a la quema de combustibles fósiles, actividades industriales y el uso en la agricultura de correctivos, fertilizantes y residuos orgánicos, entre ellos con especial preocupación, los lodos albañales (LA). ${ }^{2,3}$ Conocer el comportamiento del $\mathrm{Cd}$ en el suelo es de vital importancia para la evaluación de impactos ambientales provocados por actividades antropogénicas, fundamentalmente aquellas relacionadas con la actividad agrícola.

La magnitud del impacto ambiental que puede causar el LA adicionado al suelo está relacionada directamente con sus propiedades y las alteraciones químicas y físicas que provoca en el suelo, en especial, los factores que determinan su capacidad para inmovilizar el Cd. Alteraciones de las propiedades químicas y químico-físicas de suelos tratados con LA han sido reportadas en diferentes trabajos. ${ }^{4-7}$ La proporción de $\mathrm{Cd}$ en el suelo presente en la forma disponible es preocupante debido al peligro que puede representar para el ambiente y la salud humana. Varios métodos de extracción química han sido usados para cuantificar elementos traza disponible en suelo, ${ }^{8}$ sin embargo, de acuerdo con Oliveira, ${ }^{5}$ la selección de un extractor químico para evaluar disponibilidad de elementos inorgánicos en suelo es una tarea compleja, porque supone el carácter multielemental y, al mismo tiempo, una elevada capacidad de diagnosticar la disponibilidad de esos elementos para varias especies vegetales cultivadas en diferentes tipos de suelos.

Una forma alternativa de cuantificar directamente el $\mathrm{Cd}$ fitodisponible en suelo es mediante el método isotópico del valor

*e-mail: falvarez@cena,usp.br
$L$ que utiliza plantas que crecen en el suelo previamente marcado con un isótopo, estable $\left({ }^{111} \mathrm{Cd}\right)$ o radioactivo $\left({ }^{109} \mathrm{Cd}\right) .{ }^{9,10} \mathrm{La}$ relación entre la composición isotópica o la radioactividad específica de la solución utilizada para marcar el suelo y la encontrada en la planta, suministra el valor $L$. Este método puede ser más apropiado para determinar fitodisponibilidad de elementos en suelo porque utiliza las plantas como medio extractor, contempla tanto los procesos químico-físicos que suceden en el suelo como los procesos fisiológicos y reacciones que ocurren en la rizosfera de las plantas. El método ha sido utilizado para cuantificar la disponibilidad de diferentes elementos. Inicialmente, fue utilizado para evaluar fósforo ${ }^{10}$ y recientemente ha sido extendido para niquel, ${ }^{11}$ cadmio y zinc. ${ }^{12}$

El presente trabajo tuvo por objetivo determinar el efecto del $\mathrm{pH}$ del suelo sobre la fitodisponibilidad de $\mathrm{Cd}$ en un Xanthic Ferralsol $^{13}$ (Latossolo Vermelho Amarelo) ${ }^{14}$ tratado con LA mediante el método isotópico del valor $L$ y comparación con la disponibilidad estimada por el extractor DTPA de $\mathrm{pH}$ 7,3.

\section{PARTE EXPERIMENTAL}

El trabajo fue realizado en casa de vegetación, del Departamento de Nutrición Mineral de Plantas del CENA/USP, Piracicaba, São Paulo, en muestra de tierra recolectada de la camada superficial a la profundidad de $0-20 \mathrm{~cm}$ de un suelo Xanthic Ferralsol ${ }^{13}$ (Latossolo Vermelho-Amarelo). ${ }^{14}$ La tierra fue secada al aire y pasada por tamiz de malla de $5 \mathrm{~mm}$ y homogeneizada. Una muestra de la tierra fue pasada por tamiz de malla de $2 \mathrm{~mm}$ para la realización de los análisis de caracterización física ${ }^{15}$ y química. ${ }^{16,17}$ Los resultados de esos análisis están presentados en la tabla 1. De acuerdo con los criterios de interpretación de los análisis de suelo para fines de fertilidad, ${ }^{18}$ que establece los términos de muy bajo, bajo, medio, alto y muy alto para calificar el estado de los diferentes parámetros que miden la fertilidad, el suelo utilizado, con contenido de arcilla entre 250 e $340 \mathrm{~g} \mathrm{~kg}^{-1}$, es de textura medio arcillosa, con 
$\mathrm{pH} \leq 4,3$ era de acidez muy alta, justificada por la baja saturación en bases intercambiables ( $\mathrm{Ca}, \mathrm{Mg}$ y $\mathrm{K}$ ) la saturación por aluminio en nivel medio y la concentración de $\mathrm{Mn}$ alta. El $\mathrm{P}$ disponible era muy bajo, el Ca y $\mathrm{Mg}$ intercambiables bajos y el $\mathrm{K}$ en nivel medio de concentración (Tabla 1). El contenido total de elementos potencialmente tóxicos (EPT) como Ni $\left(8,1 \mathrm{mg} \mathrm{kg}^{-1}\right), \mathrm{Zn}\left(32 \mathrm{mg} \mathrm{kg}{ }^{-1}\right)$, $\mathrm{Cd}\left(0,17 \mathrm{mg} \mathrm{kg}^{-1}\right), \mathrm{Cu}\left(15 \mathrm{mg} \mathrm{kg}^{-1}\right)$ y $\mathrm{Pb}\left(9,9 \mathrm{mg} \mathrm{kg}^{-1}\right)$, determinado por el método EPA-3051 adoptado por la CETESB ${ }^{19}$ para la obtención de los valores de referencia y medidos por espectrometría de emisión con fuente de plasma inducida, son inferiores a los valores de referencia de calidad emitido por la $\mathrm{CETESB}^{20}$ y por tanto el suelo no estaba contaminado por esos metales.

Tabla 1. Características del suelo y del lodo albañal utilizados

\begin{tabular}{|c|c|c|c|c|}
\hline & Suelo & & Lodo & \\
\hline $\mathrm{pH}\left(\mathrm{CaCl}_{2}\right)$ & 4,0 & & 2,8 & \\
\hline Materia orgánica & 16,0 & $\left(\mathrm{~g} \mathrm{~kg}^{-1}\right)$ & 348 & $\left(\mathrm{~g} \mathrm{~kg}^{-1}\right)$ \\
\hline $\mathrm{P}$ & 6,0 & $\left(\mathrm{mg} \mathrm{dm}^{-3}\right)$ & 5,5 & $\left(\mathrm{~g} \mathrm{~kg}^{-1}\right)$ \\
\hline $\mathrm{K}$ & 2,6 & $\left(\mathrm{mmol} \mathrm{dm}^{-3}\right)$ & 1,0 & $\left(\mathrm{~g} \mathrm{~kg}^{-1}\right)$ \\
\hline $\mathrm{Ca}$ & 13,0 & $\left(\mathrm{mmol} \mathrm{dm}^{-3}\right)$ & 10,8 & $\left(\mathrm{~g} \mathrm{~kg}^{-1}\right)$ \\
\hline $\mathrm{Mg}$ & 4,0 & $\left(\mathrm{mmol} \mathrm{dm}^{-3}\right)$ & 1,7 & $\left(\mathrm{~g} \mathrm{~kg}^{-1}\right)$ \\
\hline $\mathrm{Al}^{3+}$ & 8,0 & $\left(\mathrm{mmol}_{\mathrm{c}}^{\mathrm{c}} \mathrm{dm}^{-3}\right)$ & 13,10 & $\left(\mathrm{~g} \mathrm{~kg}^{-1}\right)$ \\
\hline $\mathrm{H}^{+}+\mathrm{Al}^{3+}$ & 64,0 & $\left(\mathrm{mmol}_{\mathrm{c}}^{\mathrm{c}} \mathrm{dm}^{-3}\right)$ & - & \\
\hline SB & 19,6 & $\left(\mathrm{mmol}_{\mathrm{c}}^{\mathrm{c}} \mathrm{dm}^{-3}\right)$ & - & \\
\hline $\mathrm{CIC}$ & 83,6 & $\left(\mathrm{mmol}_{\mathrm{c}}^{\mathrm{c}} \mathrm{dm}^{-3}\right)$ & - & \\
\hline $\mathrm{V}$ & 23,0 & $(\%)$ & - & \\
\hline $\mathrm{m}$ & 29,0 & $(\%)$ & - & \\
\hline $\mathrm{Cu}$ & 1,0 & $\left(\mathrm{mg} \mathrm{kg}^{-1}\right)$ & 471,8 & $\left(\mathrm{mg} \mathrm{kg}^{-1}\right)$ \\
\hline $\mathrm{Fe}$ & 33,0 & $\left(\mathrm{mg} \mathrm{kg}^{-1}\right)$ & 22,4 & $\left(\mathrm{~g} \mathrm{~kg}^{-1}\right)$ \\
\hline Mn & 18,1 & $\left(\mathrm{mg} \mathrm{kg}^{-1}\right)$ & 925,5 & $\left(\mathrm{mg} \mathrm{kg}^{-1}\right)$ \\
\hline $\mathrm{Zn}$ & 2,3 & $\left(\mathrm{mg} \mathrm{kg}^{-1}\right)$ & 1,96 & $\left(\mathrm{~g} \mathrm{~kg}^{-1}\right)$ \\
\hline $\mathrm{Ni}$ & 0,06 & $\left(\mathrm{mg} \mathrm{kg}^{-1}\right)$ & 92,0 & $\left(\mathrm{mg} \mathrm{kg}^{-1}\right)$ \\
\hline $\mathrm{Cd}$ & 0,02 & $\left(\mathrm{mg} \mathrm{kg}^{-1}\right)$ & 47,4 & $\left(\mathrm{mg} \mathrm{kg}^{-1}\right)$ \\
\hline $\mathrm{Pb}$ & 0,73 & $\left(\mathrm{mg} \mathrm{kg}^{-1}\right)$ & 174,1 & $\left(\mathrm{mg} \mathrm{kg}^{-1}\right)$ \\
\hline $\mathrm{Cr}$ & - & & 191,6 & $\left(\mathrm{mg} \mathrm{kg}^{-1}\right)$ \\
\hline $\mathrm{Na}$ & - & & 0,91 & $\left(\mathrm{~g} \mathrm{~kg}^{-1}\right)$ \\
\hline Arena & 660 & $\left(\mathrm{~g} \mathrm{~kg}^{-1}\right)$ & & \\
\hline Limo & 60 & $\left(\mathrm{~g} \mathrm{~kg}^{-1}\right)$ & & \\
\hline Arcilla & 280 & $\left(\mathrm{~g} \mathrm{~kg}^{-1}\right)$ & & \\
\hline Textura & \multicolumn{2}{|c|}{ Medio arcillosa } & - & \\
\hline
\end{tabular}

SB: suma de bases intercambiables. CIC: capacidad de intercambio catiónico. V: \% de saturación por bases. m: \% de saturación con aluminio. nd: no detectado

El lodo albañal utilizado denominado "Jundfertil", proveniente de digestión anaeróbica y fermentación sin adición de cal, fue obtenido en la Compañía de Saneamiento de la ciudad de Jundiaí, SP. Este material fue secado, molido y pasado por tamiz con malla de $2 \mathrm{~mm}$ y su composición química (Tabla 1) fue analizada por las metodologías descritas por Camargo et al. ${ }^{16}$ Boaventura et al. ${ }^{21} \mathrm{y}$ Kiehl. ${ }^{22} \mathrm{El} \mathrm{pH}$ era ácido, lo que confirma que en el proceso de obtención e higienización no fue utilizado cal u otro tipo de material neutralizante. El bajo contenido de $\mathrm{Ca}$ en comparación con valores reportados de lodos con $\mathrm{pH}$ neutro o alcalino ${ }^{23}$ en que el proceso de higienización fue realizado con adición de cal, corrobora también que ese lodo no fue tratado con cal. El contenido de EPT en este lodo (Tabla 1) es inferior a los valores permitidos por la Norma de la CETESB ${ }^{24}$ para uso agrícola.

El experimento fue realizado adoptando un diseño completamente aleatorizado con tres repeticiones, siendo las unidades experimentales compuestas por macetas plásticas de 2,5 L. Para el estudio del efecto de la acidez del suelo sobre la fitodisponibilidad del Cd en suelo tratado con LA, los tratamientos siguieron un modelo factorial $3 \times 5$ constituido por tres valores de $\mathrm{pH}$, medidos en $\mathrm{CaCl}_{2}$ 0,01 mol L-1 , 4,0, 4,9 y 5,8 con cinco dosis de LA equivalentes a $0,15,30,45$ y $60 \mathrm{Mg} \mathrm{ha}^{-1}$ (base seca). Las dosis de LA fueron establecidas cuidando de no generar residuos contaminados, para lo cual, se consideró que la mayor dosis no alcanzara el límite máximo permitido para el $\mathrm{Cd}$ en área agrícola de protección máxima (3 mg kg-1), por ser este el elemento más limitante (considerando apenas los EPT) de acuerdo con la Norma de la Compañía de Tecnología de Saneamiento Ambiental ${ }^{20}$ vigente en el estado de São Paulo. Con las dosis de LA utilizadas en el presente trabajo, 0, $15,30,45$ y $60 \mathrm{Mg} \mathrm{ha}^{-1}$, el contenido total de Cd en las muestras de suelo de cada tratamiento fue de $0,17,0,52,0,87,1,22$ y $1,57 \mathrm{mg}$ $\mathrm{kg}^{-1}$, respectivamente.

El pH original de la tierra era 4,0 y el ajuste a valores aproximados de 5,0 y 6,0 fue realizado mediante incubación por 30 días con aplicación de mezcla de carbonatos de calcio y magnesio, de grado analítico, con relación molar Ca:Mg de 4:1. La cantidad de mezcla de carbonatos a aplicar fue calculada de la ecuación de correlación obtenida de una curva de encalado previamente obtenida. El pH alcanzado fue de 4,9 y 5,8. Seguidamente fue colocado $2 \mathrm{~kg}$ de tierra en macetas recubiertas internamente con saco plástico, se adicionó las dosis preestablecidas de LA, homogeneizó y se incubó nuevamente por 30 días en la casa de vegetación.

Para identificar las alteraciones químicas provocadas por el LA en la tierra, una muestra de esta fue retirada de cada tratamiento, la cual fue secada al aire y pasada por tamiz con abertura de malla de $2 \mathrm{~mm}$ y determinados el $\mathrm{Cd}, \mathrm{Zn}$ y $\mathrm{Mn}$ disponible con el extractor DTPA $^{25}$ de $\mathrm{pH} 7,3, \mathrm{pH}$ en $\mathrm{CaCl}_{2} 0,01 \mathrm{~mol} \mathrm{~L}^{-1}$, acidez potencial $(\mathrm{H}+\mathrm{Al})$, conductividad eléctrica $(\mathrm{CE})$, materia orgánica, fósforo disponible, $\mathrm{K}, \mathrm{Ca}$ y $\mathrm{Mg}$ intercambiables, suma de bases (SB) y capacidad de intercambio de cationes (CIC), según métodos descritos en Raij et al. ${ }^{17} \mathrm{La}$ interpretación de los resultados de los análisis químicos del suelo sobre fertilidad fueron realizados siguiendo los criterios establecidos en Raij et al.. ${ }^{18}$

La razón isotópica del Cd en las muestras de suelo de todos los tratamientos fue alterada por la adición de $100 \mathrm{~mL}$ de una solución que contenía $4 \mu \mathrm{g}$ de $\mathrm{Cd}\left(\mathrm{CdCl}_{2}\right)$ con una actividad de $148 \mathrm{kBq}(4$ $\mu \mathrm{Ci})$ de ${ }^{109} \mathrm{Cd}$ en cada maceta. La influencia de la solución de $\mathrm{Cd}$ adicionada sobre la cantidad total del metal contenido en el suelo fue pequeña, aumentando en $0,1 \%$ en el tratamiento con dosis de $60 \mathrm{Mg} \mathrm{ha}^{-1}$ de LA y $1,17 \%$ en el tratamiento sin LA. Dos días después fue realizada la homogeneización de la tierra de cada maceta y se dejó incubar por 30 días. Todas las incubaciones fueron realizadas manteniendo la humedad del suelo a $70 \%$ de la capacidad de retención de agua, la que fue controlada por el peso medido en balanza y la reposición del agua evaporada cuando fue necesario.

Considerando que la concentración de potasio en el LA es baja, todos los tratamientos recibieron $150 \mathrm{mg} \mathrm{kg}^{-1}$ del elemento en la forma de cloruro de grado analítico.

El tratamiento de referencia ( $0 \mathrm{Mg} \mathrm{ha}^{-1}$ de LA) recibió fertilización nitrogenada, dosis de $50 \mathrm{mg} \mathrm{kg}^{-1}$, en el momento de la siembra y a los 15 días de la germinación todos los tratamientos recibieron $150 \mathrm{mg} \mathrm{kg}^{-1}$. Para evitar variación del $\mathrm{pH}$ del suelo por la fertilización nitrogenada, se utilizó una mezcla de 79,85\% de $\mathrm{N}$ en la forma de nitrato de calcio y $20,15 \%$ en la forma de sulfato de amonio siguiendo la recomendación dada por Raij et al.. ${ }^{18} \mathrm{Al}$ tratamiento de referencia también se adicionó $0,5 \mathrm{mg} \mathrm{kg}^{-1}$ de $\mathrm{Cu}$ (sulfato de cobre); 0,5 mg kg-1 de B (ácido bórico); $8,1 \mathrm{mg} \mathrm{kg}^{-1} \mathrm{de}$ Mn (sulfato de manganeso) y $0,2 \mathrm{mg} \mathrm{kg}^{-1}$ de Mo (molibdato de amonio), todos en la forma de reactivos de calidad analítica.

En cada maceta fue sembrado 5 semillas de maíz (Zea mais L.) 
híbrido P30F80 y a los 5 días de la germinación fue realizado un raleo y dejado 2 plantas por maceta. A los 40 días de la germinación la parte aérea de las plantas (hojas y tallos) fue cortada aproximadamente a $1 \mathrm{~cm}$ de altura de la superficie de la tierra, lavadas con agua desionizada de conductividad $0,6 \mu \mathrm{S} \mathrm{cm}^{-1}$, secadas en estufa a $65{ }^{\circ} \mathrm{C}$, pesadas y molidas.

La radioactividad del ${ }^{109} \mathrm{Cd}$, medida en $2 \mathrm{~g}$ de muestra de material vegetal seco dispuesto en frasco plástico de $23 \mathrm{~mm}$ de diámetro, fue realizada por espectrometría gamma de alta resolución en analizador multicanal acoplado a un detector semiconductor de $\mathrm{Ge}(\mathrm{Li})$ hiperpuro utilizando el fotopico de $88 \mathrm{keV}$ de la radiación gamma emitida por el radioisótopo ${ }^{109 \mathrm{~m}} \mathrm{Ag}$ (media vida $=40 \mathrm{~s}$ ) producido en la desintegración del ${ }^{109} \mathrm{Cd}$ (media vida $=450$ días). Las mediciones fueron realizadas con un $95 \%$ de confiabilidad, para lo cual fue fijado un tiempo de medición de $1000 \mathrm{~s}$.

Para el análisis de Cd en las muestras de material vegetal fue realizada digestión nitro-perclórica siguiendo el método descrito en Bataglia et al. ${ }^{26}$ con modificación de la relación peso de planta/ volumen de dilución $(\mathrm{g} / \mathrm{mL})$ de $0,5 / 50$ para $1 / 25$ con objetivo de garantizar en la solución una concentración de Cd superior al límite de detección del método analítico. Los análisis fueron realizados por espectrometría de emisión con fuente de plasma inducida en un espectrómetro Perkin Elmer modelo 3000DV. Para el control de la fiabilidad de los análisis fue utilizado muestras de referencia certificadas de suelo (National Institute of Standards \& Technology SRM 2710) y de arroz (National Institute of Environmental Science NIES № $10_{C}$ ) cuyos resultados obtenidos fueron concordantes con los valores certificados de acuerdo al estadígrafo t de Students al nivel de confianza de $95 \%$.

El cálculo del valor L fue realizado mediante la ecuación siguiente: ${ }^{27}$

valor $L=M\left(\frac{A_{1}}{A_{2}}-1\right)$

donde: $M=$ cantidad de $\mathrm{Cd}$ adicionado con la solución radioactiva a la tierra $\left(2 \mu \mathrm{g} \mathrm{kg}^{-1}\right) ; A_{1}=$ actividad específica del Cd en la solución adicionada a la tierra $\left(\mathrm{cpm} \mu \mathrm{g}^{-1}\right) ; A_{2}=$ actividad específica del $\mathrm{Cd}$ en la planta (cpm $\left.\mu \mathrm{g}^{-1}\right)$.

Con las actividades específicas de las plantas y las Ecuaciones 2 y 3 fue determinado el porcentaje de $\mathrm{Cd}$ en la planta proveniente de la tierra (\%Cdppt) y del LA (\%CdppLA):

$\% C d p p t=\left(\frac{a_{L A}}{a_{0}}\right) \times 100$

$\% C d p p L A=(100-\% C d p p t)$

donde: $a_{L A}=$ actividad específica del $\mathrm{Cd}\left(\mathrm{cpm} \mu \mathrm{g}^{-1}\right)$ en las plantas de tratamientos con $n=15,30,45$ y $60 \mathrm{Mg} \mathrm{ha}^{-1}$ de LA; $a_{0}=$ actividad específica del $\mathrm{Cd}$ en las plantas del tratamiento de referencia sin adición de LA.

La interpretación de los resultados fue realizada mediante el análisis de varianza y de regresión con el programa SAS. ${ }^{28}$ Siempre que el análisis de varianza (prueba F) detectó diferencias significativas entre tratamientos, se realizó el análisis de comparación múltiple de los valores medios de los tratamientos de los resultados a través de la prueba de Tukey a 5\% de significación y evaluaciones por medio de regresiones lineales simples y polinomiales. La fitodisponibilidad de $\mathrm{Cd}$ fue analizada tanto, a través de correlación entre los resultados obtenidos del valor $L$, como de la extracción de Cd por DTPA con la cantidad del elemento acumulada en la parte aérea de las plantas.

\section{RESULTADOS Y DISCUSIÓN}

Las dosis crecientes de LA ácido adicionadas a las muestras de suelo provocó disminución del $\mathrm{pH}$ actual y aumentos de la acidez potencial $(\mathrm{H}+\mathrm{Al}$ ) (Figura 1), la materia orgánica, $\mathrm{P}$ disponible, conductividad eléctrica, $\mathrm{Ca}, \mathrm{Mg}$ intercambiables y la capacidad de intercambio de cationes (CIC) (Figura 2).

El valor de las pendientes significativas (b) de las ecuaciones de regresión simples, en la variación del pH (Figura 1), muestra que las variaciones de $\mathrm{pH}$ fueron mayores en las muestras de suelos de $\mathrm{pH} 4,9$ y 5,8 que en la de $\mathrm{pH} 4,0$. En la tierra de $\mathrm{pH} 4,9$, la acidez pasó de alta para muy alta $(\mathrm{pH} 3,9)$ y en la tierra de $\mathrm{pH} 5,8$ pasó de acidez baja para muy alta $(\mathrm{pH} 4,1)$ según los criterios establecidos

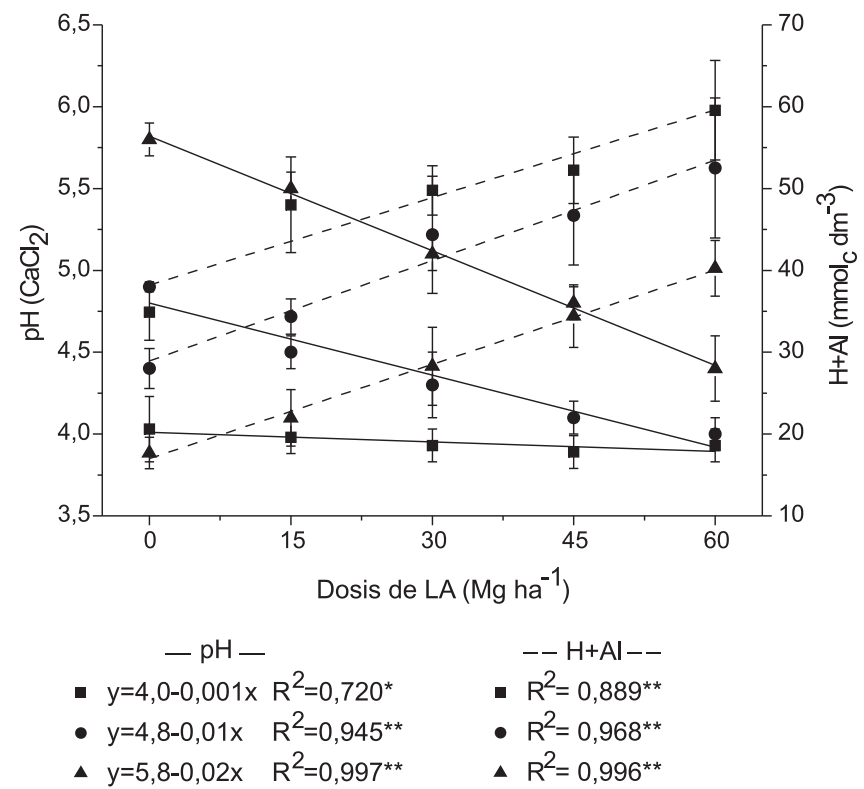

Figura 1. Alteraciones del $\mathrm{pH}$ y la acidez potencial del suelo $(H+A l)$ con las dosis de LA en suelo de $\mathrm{pH} \mathbf{\square}$ 4,0, 4,9 y $\mathbf{\Delta} 5,8$. * y ** significativo a $5 \%$ y $1 \%$ de probabilidad, respectivamente, por la prueba $F$

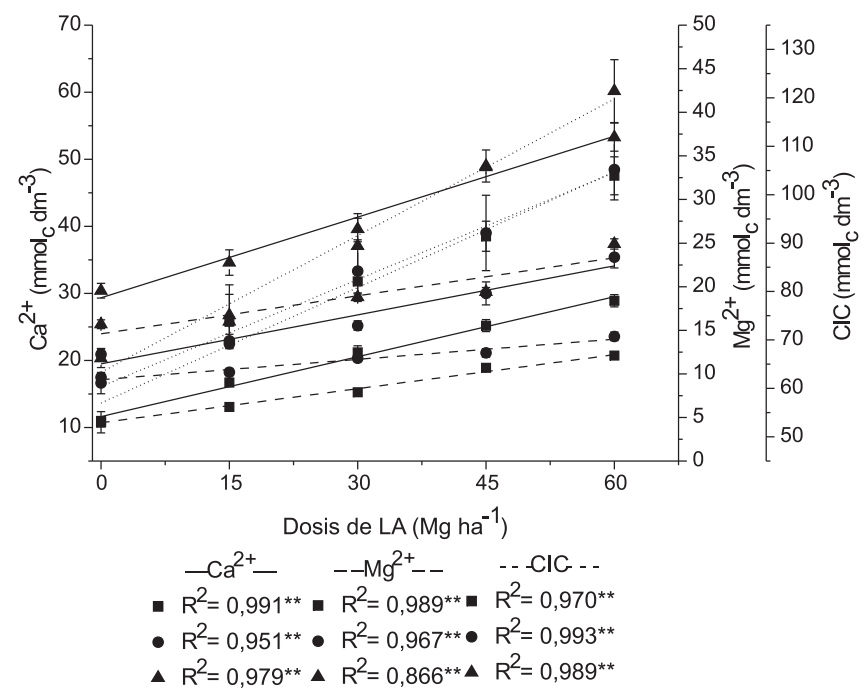

Figura 2. Alteraciones del Ca y Mg intercambiables y la capacidad de intercambio de cationes, con las dosis de LA en suelos de diferente $p H$ 4,0, $\bullet, 9$ y $\boldsymbol{\Delta} 5,8$. * y ** significativo a $5 \%$ y $1 \%$ de probabilidad, respectivamente, por la prueba $F$ 
en Raij. ${ }^{18}$ En la tierra de $\mathrm{pH} 4,0$ no hubo modificación significativa del $\mathrm{pH}(4,0$ a 3,8$)$ permaneciendo en acidez muy alta ${ }^{18}$ debido a la falta de agentes neutralizadores y la acción buffer de las arcillas y MO del suelo que regulan las variaciones de la acidez. ${ }^{29}$ Las alteraciones que ocurrieron en el $\mathrm{pH}$ de los suelos que tuvieron el $\mathrm{pH}$ ajustado con adición de carbonatos de $\mathrm{Ca}$ y $\mathrm{Mg}$ se deben a la neutralización gradual de iones $\mathrm{HO}^{-}$que fueron suministrados con los carbonatos de $\mathrm{Ca}$ y $\mathrm{Mg}$. Otra causa de la acidificación del suelo por el LA podría ser la mineralización de la materia orgánica que también aporta iones $\mathrm{H}^{+}$. Acidificación de suelo tratado con LA de pH 6,3 debido a la mineralización de la materia orgánica del suelo fue reportado por Simonete. ${ }^{30} \mathrm{La}$ acidez potencial $(\mathrm{H}+\mathrm{Al})$ también aumentó linealmente con las dosis de LA (Figura 1). El mayor incremento ocurrió en la tierra de $\mathrm{pH}$ 4,0 donde no hay agentes neutralizadores de la acidez aportada por el lodo (Tabla 1) mientras que, en las tierras de $\mathrm{pH} 4,9$ y 5,8 , el incremento de la acidez potencial fue inferior debido a la neutralización parcial de la acidez y la acción buffer del suelo.

La CE aumentó con las dosis de LA de 0,06 a $0,52 \mathrm{mS} \mathrm{cm}^{-1}$ en las tierras de $\mathrm{pH} 4,0$ y 4,9 y de 0,09 a $0,65 \mathrm{mS} \mathrm{cm}^{-1}$ en la tierra de pH 5,8 debido al aporte de los elementos que contiene el LA, fundamentalmente de Fe, Al, Ca, P, Mg y EPT $(\mathrm{Ni}+\mathrm{Cu}+\mathrm{Zn}+\mathrm{Mn}+$ $\mathrm{Cd}+\mathrm{Pb}+\mathrm{Cr}$ ) (Tabla1). De acuerdo a las dosis de lodo utilizadas, las cantidades adicionadas de cada elemento por $\mathrm{kg}$ de tierra variaron de 168 a 672,98 a 393, 81 a 324, 41 a $161,12,8$ a 51,0 y 28 a 115 $\mathrm{mg}$, respectivamente. Los aumentos de la $\mathrm{CE}$, en relación al tratamiento testigo ( $0 \mathrm{Mg} \mathrm{ha}^{-1}$ de LA), fueron de 785,876 y $812 \%$ en las tierras de $\mathrm{pH} 4,0,4,9$ y 5,8 respectivamente, sin embargo, todos fueron inferiores al valor limite de $2,0 \mathrm{mS} \mathrm{cm} \mathrm{cm}^{-1}$ en extracto de saturación para el cual el cultivo de maíz es sensible. ${ }^{29}$ El incremento de la CE es indicativo del aumento de la concentración de iones en la solución del suelo y su fuerza iónica, la que tiene importante influencia en la adsorción y desorción de iones en la fase sólida del suelo y consecuentemente sobre la disponibilidad y fitodisponibilidad de EPT. La disminución de la adsorción de Cd en 17 Latossolos de Brasil (no tratados con lodo) con el aumento de la fuerza iónica fue reportado por Pierangeli et al.. ${ }^{31}$

De acuerdo con el aporte de MO por el LA utilizado, el contenido en las muestras de suelo debió variar de medio para alto en las dosis de 45 y $60 \mathrm{Mg} \mathrm{ha}^{-1}$ pero, se observó que se mantuvo constante dentro del nivel medio, de acuerdo con los criterios establecidos en Raij et al., ${ }^{18}$ debido a la mineralización durante el proceso de incubación de la tierra con el LA. La mineralización de la materia orgánica durante el proceso de incubación, en las muestras de suelo tratadas con LA, fue de 15 y $27 \%$ entre la menor y mayor dosis de LA, respectivamente. El contenido de MO, con independencia del $\mathrm{pH}$ de la tierra, varió linealmente $\left(\mathrm{R}^{2}=0,970\right)$ de $30 \mathrm{~g} \mathrm{~kg}^{-1}$ en el tratamiento sin aplicación de LA a $46 \mathrm{~g} \mathrm{~kg}^{-1}$ en la mayor dosis de lodo.

En la tierra de pH 4,0 el contenido de $\mathrm{K}$, según los criterios establecidos en Raij, ${ }^{18}$ varió de medio, 2,66 $\pm 0,24 \mathrm{mmol}_{\mathrm{c}} \mathrm{dm}^{-3}$, para alto, 3,34 $\pm 0,24 \mathrm{mmol}_{\mathrm{c}} \mathrm{dm}^{-3}$, mientras que, en las muestras de suelo de $\mathrm{pH} 4,9$ y 5,8 se mantuvo dentro de la categoría de contenido medio, con variación de 2,6 $\pm 0,24$ a 2,96 $\pm 0,25 \mathrm{mmol}_{\mathrm{c}} \mathrm{dm}^{-3}$. Ese comportamiento puede ser explicado por la mayor fuerza de retención del elemento en el complejo de cambio catiónico de la tierra en la medida que el $\mathrm{pH}$ aumenta y hay mayor carga negativa sobre la superficie de las partículas coloidales.

$\mathrm{El} \mathrm{Ca} \mathrm{y} \mathrm{Mg} \mathrm{intercambiables,} \mathrm{aumentaron} \mathrm{linealmente} \mathrm{con} \mathrm{las}$ dosis de LA (Figura 2). La concentración de Ca y Mg intercambiable, con la mayor dosis de LA, en la tierra de $\mathrm{pH} 4,0$ tuvo un aumento de 168 y $164 \%$, de 69 y $49 \%$ en la tierra de $\mathrm{pH} 4,9$ y de 75 y $58 \%$ en la tierra de $\mathrm{pH} 5,8$, respectivamente. El menor incre- mento relativo de $\mathrm{Ca}$ y $\mathrm{Mg}$ intercambiables en las tierras de $\mathrm{pH} 4,9$ y 5,8 se debió a la mayor cantidad de Ca y Mg que contenian como consecuencia del encalado realizado para el ajuste de la acidez, que elevó el $\mathrm{Ca}$ intercambiable de un nivel bajo para medio. Posteriormente, con la adición de lodo, la cantidad de Ca intercambiable pasó de la clasificación de nivel bajo para medio en las muestras de suelo de $\mathrm{pH} 4,0$ y 4,9 y de medio para alto en la de $\mathrm{pH} 5,8$. El $\mathrm{Mg}$ intercambiable inicial del suelo (Tabla 1) era medio (4 mmol $\left.\mathrm{dm}^{-3}\right)^{18}$ y con la adición de $\mathrm{MgCO}_{3}$ pasó para alto en las muestras de suelo de pH 4,9 y 5,8. En la tierra de pH 4,0 la aplicación de LA elevó el $\mathrm{Mg}$ intercambiable de medio para alto mientras que, en las tierras de $\mathrm{pH}$ 4,9 y 5,8 se mantuvo en la categoría de contenido alto independientemente de la aplicación de LA.

La capacidad de intercambio de cationes (CIC) fue linealmente creciente con las dosis de LA (Figura 2) y los mayores valores se observaron en la tierra de $\mathrm{pH}$ 5,8 (Figura 2) lo que se justifica por el $\mathrm{pH}$ de la misma. ${ }^{4-6}$ Varios estudios han corroborado el aumento de la CIC con la aplicación de lodo. ${ }^{4,6} \mathrm{El}$ aumento de la CIC con las dosis de LA puede ser atribuido al incremento de la materia orgánica la cual presenta sitios activos constituidos por grupos funcionales que dependiendo del $\mathrm{pH}$ poseen capacidad variable de ligar e intercambiar iones. ${ }^{29}$ La mayor influencia de la materia orgánica del lodo en las propiedades químicas del suelo está en la alteración del complejo coloidal de este. En función del estado coloidal de la MO, con elevada superficie específica, esta presenta elevada capacidad de cambio catiónico al mismo tiempo que es dependiente del $\mathrm{pH}$ del medio. ${ }^{32}$ A pesar de la alta correlación significativa entre dosis de lodo y la CIC (Figura 2), se resalta que, como observado por Oliveira et al., ${ }^{5}$ la determinación de la CIC por la suma de bases intercambiables y la acidez potencial, conforme fue realizado en el presente trabajo, podría haber ocasionado la sobrestimación de ese índice por causa de los aportes de $\mathrm{Ca}$ y $\mathrm{Mg}$ contenidos en el lodo.

El P disponible de los tratamientos testigo $\left(0 \mathrm{Mg} \mathrm{ha}^{-1}\right.$ de LA) mostró una reducción de 34 y $50 \%$, en las tierras de $\mathrm{pH} 4,9$ y 5,8, respectivamente, en relación al valor original, con el aumento del $\mathrm{pH}$. Factores como el $\mathrm{pH}$, materia orgánica, cationes divalentes ligados a las arcillas y actividad microbiana regulan la disponibilidad de fosfatos en el suelo. ${ }^{29}$ Esa reducción podría estar dada por efecto catiónico de los iones $\mathrm{Ca}^{2+}$ adicionado a la tierra con el carbonato. El Ca desplaza los iones $\mathrm{Al}^{3+}$ ligados a las arcillas, estos se hidrolizan formando hidróxido de aluminio que reacciona con los iones fosfato y precipitan, ${ }^{29}$ o puede ocurrir asociación del fosfato con la materia orgánica por medio de intercambio aniónico con grupos $\mathrm{OH}$ de la materia orgánica o aún la formación de complejos coloidales de la materia orgánica asociada a óxidos de hierro e aluminio con el fosfato que reducen su disponibilidad. ${ }^{33,34}$ Hubo aumentos significativos del $\mathrm{P}$ disponible con las dosis de LA, que según los criterios establecidos en Raij, ${ }^{18}$ fueron desde bajo, $7 \mathrm{mg} \mathrm{dm}^{-3}$, en la tiera de $\mathrm{pH} 4,0$ y de muy bajo, 4,7 $\mathrm{mg} \mathrm{dm}^{-3}$ en la tierra de $\mathrm{pH} 4,9$ y $3,5 \mathrm{mg}$

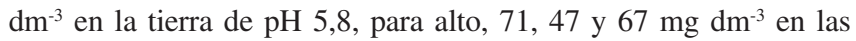
tierras de $\mathrm{pH} 4,0,4,9$ y 5,8, respectivamente, con la dosis de $60 \mathrm{Mg}$ $\mathrm{ha}^{-1}$ de LA. Además de ser fuente de $\mathrm{P}$, el lodo puede actuar en la disminución de la adsorción del elemento en el suelo, debido a la materia orgánica del mismo que compite con el fosfato por los sitios de adsorción. ${ }^{29}$

Las alteraciones químicas provocadas en la tierra por el lodo se reflejan en la fitodisponibilidad del Cd. La fitodisponibilidad de $\mathrm{Cd}$, determinada por el valor $L$ aumentó linealmente con las dosis de LA (Tabla 2). Los aumentos relativos al tratamiento referencia fueron, de la menor a la mayor dosis, de 2 a 7 veces en las tierras de $\mathrm{pH} 4,0$ y 4,9 y de 1 a 4 veces en la tierra de $\mathrm{pH} 5,8$. Los menores incrementos en esta última tierra estuvieron condicionados por el incremento 
Tabla 2. Fitodisponibilidad de $\mathrm{Cd}$ (Valor $L$ ) en suelo de diferente $\mathrm{pH}$ tratado con LA y correlaciones lineales con las dosis de lodo

\begin{tabular}{|c|c|c|c|c|c|c|}
\hline \multicolumn{6}{|c|}{ pH inicial de las muestras de suelo } & \\
\hline \multirow[b]{2}{*}{$\mathrm{Mg} \mathrm{ha}^{-1}$} & 4,0 & & 4,9 & & 5,8 & \\
\hline & \multicolumn{6}{|c|}{ Valor $L$ de $\mathrm{Cd}\left(\mu \mathrm{g} \mathrm{kg}^{-1}\right)$} \\
\hline 0 & 27,5 & $\mathrm{~b}$ & 22,9 & $\mathrm{~b}$ & 37,8 & $\mathrm{a}$ \\
\hline 15 & 58,8 & $\mathrm{a}$ & 66,9 & a & 46,4 & $\mathrm{~b}$ \\
\hline 30 & 90,7 & $\mathrm{a}$ & 82,2 & a & 87,6 & $\mathrm{a}$ \\
\hline 45 & 137,6 & $\mathrm{a}$ & 123,0 & $\mathrm{~b}$ & 108,3 & $\mathrm{c}$ \\
\hline 60 & 180,4 & a & 179,8 & $\mathrm{a}$ & 144,7 & $\mathrm{~b}$ \\
\hline Modelo & $\begin{array}{c}y=2,56 x \\
+22,12\end{array}$ & & $\begin{array}{c}y=2,46 x \\
+20,94\end{array}$ & & $\begin{array}{c}y=1,83 x \\
+29,83\end{array}$ & \\
\hline $\mathrm{R}^{2}$ & $0,992 * *$ & & $0,969 * *$ & & $0,970 * *$ & \\
\hline
\end{tabular}

Valores con letras iguales en las líneas no difieren a $5 \%$ por la prueba de Tukey. **Indica correlación significativa $(P \leq 0,01)$ por la prueba $F$.

de la fitodisponibilidad en el tratamiento de referencia y la disminución en los tratamientos con LA en relación a las tierras de menor $\mathrm{pH}$. El aumento del valor $\mathrm{L}$ con las dosis se explica por los aportes del LA y las alteraciones químicas que el mismo provoca en la tierra, fundamentalmente, la disminución del pH (Figura 1).

El mayor valor $L$ obtenido en el tratamiento referencia de la tierra de $\mathrm{pH}$ 5,8 indica que hubo incremento de la fitodisponibilidad de $\mathrm{Cd}$, contrario al resultado obtenido con el extractor DTPA que disminuyó (Tabla 3). Investigaciones del efecto de la acidez del suelo sobre la disponibilidad de $\mathrm{Cd}$ han mostrado que el aumento del $\mathrm{pH}$ disminuye la concentración del metal disponible por varias causas, entre ellas, mayor adsorción en los coloides inorgánicos y orgánicos, o de retención en el complejo de intercambio catiónico o la formación de compuestos de baja solubilidad, pero, es conocido que el incremento del $\mathrm{pH}$ en el suelo favorece el desarrollo de las plantas, en especial, el crecimiento y funciones fisiológicas de la raíz. ${ }^{35,36}$ El valor $L$ es calculado de acuerdo con la Ecuación 1, es decir, por la relación entre las actividades específicas del ${ }^{109} \mathrm{Cd}$ adicionada a la tierra y la encontrada en la planta, ${ }^{10,27}$ de ahí que, el aumento en el valor $L$ se debe a la disminución de la actividad específica de ${ }^{109} \mathrm{Cd}$ en la planta (mayor dilución isotópica del ${ }^{109} \mathrm{Cd}$ ). Esto indica que las plantas absorbieron $\mathrm{Cd}$ de compuestos que la solución de DTPA no extrae. Este resultado demuestra el comportamiento diferenciado entre el extractor biológico constituido por las plantas y el extractor químico DTPA, en función de las condiciones químico-físicas y biológicas del sistema en estudio y confirma que, el valor $L$ es una función integrada de los factores químico-físicos del suelo y fisiológicos de las plantas.

La disponibilidad de Cd obtenida por el método de extracción con solución DTPA pH 7,325 también fue creciente con las dosis de LA, con correlación lineal significativa (Tabla 3). Los incrementos relativos al tratamiento referencia, de la menor a la mayor dosis de LA, fueron de 3 a 11 veces en las tierras de $\mathrm{pH} 4,0$ y 4,9 y de 4 a 13 veces en la tierra de $\mathrm{pH} 5,8$. La diferencia en los incrementos entre la fitodisponibilidad y la disponibilidad se deben a las características intrínsecas, ya explicadas, que distingue cada método.

La concentración de $\mathrm{Cd}$ y el acumulado en la parte aérea de las plantas aumentaron con las dosis de LA adicionadas y disminuyeron con el pH inicial de la tierra (Tabla 4). A pesar de los incrementos ocurridos, la concentración de Cd en las plantas de todos los tratamientos fue inferior al valor de toxicidad de este elemento en plantas, reportado por Malavolta. ${ }^{1} \mathrm{El}$ mayor valor de $\mathrm{Cd}$ acumulado fue en la dosis de $60 \mathrm{Mg} \mathrm{ha}^{-1}$ de LA dado por las mayores concentraciones en las plantas en ese tratamiento y compensado por
Tabla 3. Disponibilidad de Cd (Cd-DTPA) en suelo de diferente $\mathrm{pH}$ tratado con LA y correlaciones lineales con las dosis de lodo

\begin{tabular}{lcccccc}
\hline \multicolumn{6}{c}{ Dosis de LA } & \multicolumn{5}{c}{$\mathrm{pH}$ inicial de las muestras de suelo } \\
& 4,0 & 4,9 & \\
$\mathrm{Mg} \mathrm{ha}^{-1}$ & \multicolumn{5}{c}{ Cd - DTPA $\left(\mu \mathrm{kg}^{-1}\right)$} \\
\hline 0 & 19,5 & $\mathrm{a}$ & 17,0 & $\mathrm{a}$ & 13,0 & $\mathrm{~b}$ \\
15 & 63,0 & $\mathrm{a}$ & 56,3 & $\mathrm{~b}$ & 56,0 & $\mathrm{~b}$ \\
30 & 111,0 & $\mathrm{a}$ & 102,4 & $\mathrm{~b}$ & 99,1 & $\mathrm{~b}$ \\
45 & 166,0 & $\mathrm{a}$ & 146,0 & $\mathrm{~b}$ & 145,4 & $\mathrm{~b}$ \\
60 & 197,0 & $\mathrm{a}$ & 187,4 & $\mathrm{~b}$ & 173,9 & $\mathrm{c}$ \\
\hline Modelo & $\mathrm{y}=3,05 \mathrm{x}$ & $\mathrm{y}=2,87 \mathrm{x}$ & $\mathrm{y}=2,68 \mathrm{x}$ \\
& $+19,68$ & $+15,72$ & $+17,76$ \\
$\mathrm{R}^{2}$ & $0,994 * *$ & $0,999 * *$ & $0,995^{* *}$ \\
\hline
\end{tabular}

Valores con letras iguales en las líneas no difieren a $5 \%$ por la prueba de Tukey. **Indica correlación significativa $(P \leq 0,01)$ por la prueba $F$.

Tabla 4. Concentración de Cd y acumulado en la parte aérea de las plantas cultivadas en suelo de diferente $\mathrm{pH}$ tratado con dosis de lodo albañal (LA)

\begin{tabular}{|c|c|c|c|c|c|c|c|c|}
\hline \multirow{3}{*}{$\begin{array}{l}\text { Dosis de } \\
\mathrm{LA} \\
\mathrm{Mg} \mathrm{ha}^{-1}\end{array}$} & \multicolumn{7}{|c|}{$\mathrm{pH}$ inicial de las muestras de suelo } & \\
\hline & 4,0 & & 4,9 & & & 5,8 & & \\
\hline & \multicolumn{8}{|c|}{ Concentración de $\mathrm{Cd}\left(\mu \mathrm{g} \mathrm{kg}^{-1}\right)$} \\
\hline 0 & 0,05 & e A & 0,03 & $\mathrm{e}$ & $\mathrm{B}$ & 0,03 & $\mathrm{e}$ & $\mathrm{B}$ \\
\hline 15 & 0,37 & d A & 0,22 & $\mathrm{~d}$ & $\mathrm{~B}$ & 0,10 & $\mathrm{~d}$ & $\mathrm{C}$ \\
\hline 30 & 0,69 & c A & 0,44 & $\mathrm{c}$ & $\mathrm{B}$ & 0,34 & $\mathrm{c}$ & $\mathrm{B}$ \\
\hline 45 & 1,03 & b A & 0,84 & $\mathrm{~b}$ & $\mathrm{~B}$ & 0,41 & $\mathrm{~b}$ & $\mathrm{C}$ \\
\hline \multirow[t]{2}{*}{60} & 1,25 & a A & 1,18 & $\mathrm{a}$ & A & 0,67 & $\mathrm{a}$ & $\mathrm{B}$ \\
\hline & \multicolumn{8}{|c|}{ Cd acumulado por las plantas ( $\mu$ g por maceta) } \\
\hline 0 & 0,16 & e A & 0,08 & $\mathrm{~d}$ & B & 0,07 & $\mathrm{e}$ & B \\
\hline 15 & 1,70 & d A & 1,34 & $\mathrm{c}$ & B & 0,49 & $\mathrm{~d}$ & $\mathrm{C}$ \\
\hline 30 & 2,02 & c B & 2,88 & $\mathrm{~b}$ & A & 2,08 & $\mathrm{c}$ & B \\
\hline 45 & 3,63 & b $\quad B$ & 4,56 & $\mathrm{a}$ & A & 2,77 & $\mathrm{~b}$ & $\mathrm{C}$ \\
\hline 60 & 4,57 & a A & 4,33 & $\mathrm{a}$ & A & 4,92 & $\mathrm{a}$ & A \\
\hline
\end{tabular}

Valores con letras iguales, minúsculas en las columnas y mayúsculas en las líneas dentro de cada dosis de LA, no difieren a 5\% por la prueba de Tukey.

los rendimientos de la materia seca que se mostró con variaciones en relación a las dosis de LA ajustadas a regresiones cuadráticas en las muestras de suelo de $\mathrm{pH}$ 4,9 y 5,8 y cúbica en la de $\mathrm{pH}$ 4,0 (Figura 3).

Las variaciones de los rendimientos obtenidos responden a bajos niveles de nutrientes o desordenes nutricionales y la alta acidez del suelo que causa toxicidad por aluminio en las raíces. ${ }^{29,35,36} \mathrm{~A} \mathrm{pH} \leq$ 4,5 , la toxicidad de los iones $\mathrm{H}^{+}$afecta las membranas y respiración de las raíces, ${ }^{35}$ y con excepción de los tratamientos de referencia y $15 \mathrm{Mg} \mathrm{ha}^{-1}$ de LA en la tierra de $\mathrm{pH}$ inicial 5,8, el resto de los tratamientos tuvieron $\mathrm{pH}$ de la tierra inferior al valor adecuado (pH 5,5) para el cultivo de maíz. ${ }^{35}$

El Cd acumulado por las plantas mostró correlación significativa en los tres $\mathrm{pH}$ de las muestras de suelo con el valor $L$ y las extracciones de Cd con DTPA (Tabla 5). Las altas correlaciones obtenidas confirman la validez del método isotópico del valor $L$ para determinar la fitodisponibilidad de $\mathrm{Cd}$ en suelo tratado con LA en las condiciones experimentales del presente trabajo. De igual forma, las correlaciones significativas del $\mathrm{Cd}$ acumulado en las plantas con las extracciones por DTPA y aceptando que, el valor $L$, al ser determinado a través de las propias plantas, es el mejor índi- 


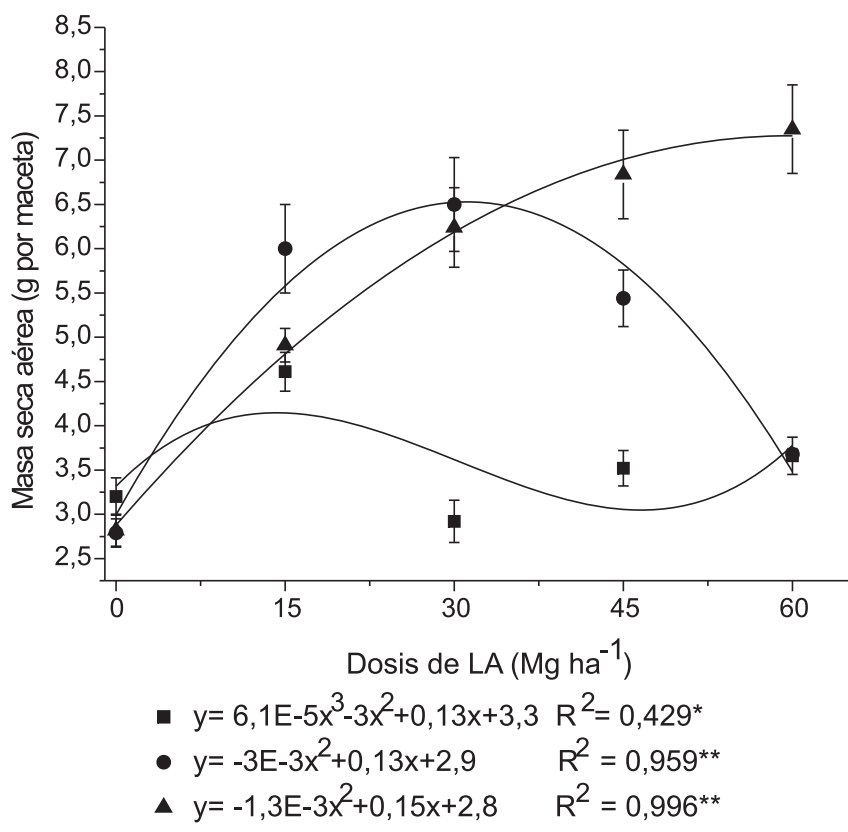

Figura 3. Rendimiento de materia seca de la parte aérea de las plantas de maíz en suelo de diferente $\mathrm{pH} \mathbf{\square}$ 4,0, 4,9 y $\mathbf{\Delta} 5,8$ tratado con dosis creciente de lodo albañal. *, ** Significativo a $5 \%$ y $1 \%$ de probabilidad, respectivamente, por la prueba $F$

Tabla 5. Coeficientes de correlación lineal entre Cd acumulado por las plantas con el valor $L$ de Cd del suelo y con el Cd extraído por DTPA (Cd-DTPA). Correlaciones entre Cd-DTPA y el valor $L$

\begin{tabular}{lccc}
\hline Método & $\mathrm{pH} \mathrm{4,0}$ & $\mathrm{pH} \mathrm{4,9}$ & $\mathrm{pH} \mathrm{5,8}$ \\
\hline Valor $L$ & $0,981 * *$ & $0,978 * *$ & $0,992 * *$ \\
Cd-DTPA & 0,999** & $0,980 * *$ & $0,937 * *$ \\
Cd-DTPA - valor $L$ & 0,984** & $0,962 * *$ & $0,954 * *$ \\
\hline
\end{tabular}

** Correlación significativa a $P \leq 0,01$, por la prueba $F$.

ce de fitodisponibilidad, entonces, las correlaciones significativas obtenidas entre la extracción de Cd con DTPA y el valor $L$ indican que el extractor químico fue un buen estimador de la fitodisponibilidad de ese elemento en suelo tratado con LA.

La fitodisponibilidad de $\mathrm{Cd}$ en suelo es condicionada por diferentes factores entre ellos, las especies de Cd presentes, textura del suelo, cantidad y calidad de la materia orgánica, capacidad de intercambio catiónico, $\mathrm{pH}$, fuerza iónica de la solución del suelo o conductividad eléctrica, competencia con otros elementos y el tipo de cultivo. ${ }^{2,3} \mathrm{El} \mathrm{Cd}$ forma compuestos menos solubles a medida que el $\mathrm{pH}$ es aumentado y por otra parte, la energía de adsorción en la fase sólida crece y disminuye la concentración del elemento en la solución del suelo. ${ }^{2,3,31,37}$ La fisiología de absorción de elementos por las plantas también tiene un papel esencial en la fitodisponibilidad y es afectada sensiblemente por el $\mathrm{pH}$ del suelo, lo que no puede ser confundido con la disponibilidad en el suelo, así por ejemplo, el aumento de la acidez incrementa la disponibilidad de elementos pero, el aumento de la concentración de $\mathrm{H}^{+}$puede disminuir la absorción de cationes debido a la competencia por ocupar los mismos sitios del cargador iónico en el mecanismo activo de absorción ${ }^{1}$ de las plantas. Con el Cd específicamente, puede ocurrir competencia de los iones $\mathrm{Zn}^{2+} \mathrm{y}$ $\mathrm{Ca}^{2+}$ que reducen la absorción de Cd por las plantas. ${ }^{1,2,38}$ La materia orgánica contribuye para la retención de metales en el suelo por su elevada capacidad de intercambio catiónico. Por otra parte, con dependencia de la calidad de la materia orgánica los metales son
Tabla 6. Porcentajes de Cd absorbidos de la tierra (\%Cdppt) y del LA (\%CdppLA) por las plantas en función de las dosis de LA y pH inicial de la tierra

\begin{tabular}{lcccc}
\hline \%* & \multicolumn{5}{c}{ Dosis de LA $\left(\mathrm{Mg} \mathrm{ha}^{-1}\right)$} \\
& 15 & 30 & 45 & 60 \\
& \multicolumn{5}{c}{$\mathrm{pH} \mathrm{4,0}$} \\
\hline Cdppt & $49 \pm 3$ & $36 \pm 3$ & $21 \pm 1$ & $16 \pm 1$ \\
CdppLA & $51 \pm 3$ & $64 \pm 3$ & $79 \pm 1$ & $84 \pm 1$ \\
\hline \multicolumn{5}{c}{$\mathrm{pH} \mathrm{4,9}$} \\
\hline Cdppt & $32 \pm 4$ & $30 \pm 2$ & $20 \pm 1$ & $14 \pm 1$ \\
CdppLA & $68 \pm 4$ & $70 \pm 2$ & $80 \pm 1$ & $86 \pm 1$ \\
\hline \multicolumn{5}{c}{$\mathrm{pH} \mathrm{5,8}$} \\
\hline Cdppt & $84 \pm 2$ & $44 \pm 2$ & $37 \pm 2$ \\
CdppLA & $16 \pm 2$ & $56 \pm 2$ & $63 \pm 2$ & $74 \pm 1$ \\
\hline
\end{tabular}

* $95 \%$ de confiabilidad.

acomplejados en la solución del suelo o en la fase sólida. ${ }^{2,3} \mathrm{La}$ fuerza iónica de la solución, que es función de la concentración de los iones y su carga presentes en la solución, tiene una gran influencia en la adsorción y consecuentemente en la disponibilidad de metales en el suelo. ${ }^{31}$

El uso del trazador isotópico ${ }^{109} \mathrm{Cd}$ permitió también conocer el origen del Cd adsorbido por las plantas. Con las Ecuaciones 2 y 3 fueron calculados los porcentajes de Cd absorbidos del que contenía la tierra (\%Cdppt) y del aportado por el LA (\%CdppLA) (Tabla 6). En función de las dosis crecientes de LA, el Cd de la tierra absorbido por las plantas disminuyó, mientras que, el Cd utilizado del LA aumentó, lo que confirma, el incremento de la disponibilidad y fitodisponibilidad tanto por el aumento del Cd con las dosis de LA como por la disminución del $\mathrm{pH}$ causada por el lodo. Por otra parte, en función del $\mathrm{pH}$ inicial de la tierra se observa un efecto contrario, cuando mayor fue el pH el \%CdppLA disminuyó y el \%Cdppt aumentó. La disminución del $\mathrm{Cd}$ absorbido del LA al aumentar el pH de la tierra puede estar influenciado por varios factores, entre ellos, por la materia orgánica del propio lodo que aumenta la capacidad de retención de $\mathrm{Cd}$ en la medida que el $\mathrm{pH}$ aumenta, la formación de complejos de baja solubilidad del Cd con la materia orgánica o aumento de la adsorción del Cd en los coloides minerales y orgánicos del suelo o del LA. La combinación de factores como el pH con la carga orgánica y su calidad regularon la disponibilidad del $\mathrm{Cd}$.

\section{CONCLUSIONES}

La adición de lodo de aguas albañales aumentó las cantidades disponibles y fitodisponibles de $\mathrm{Cd}$ en las muestras de suelo de diferente $\mathrm{pH}$.

El valor $L$ y la solución extractora de DTPA fueron eficientes en la previsión de fitodisponibilidad de $\mathrm{Cd}$ en suelo tratado con lodo albañal en este trabajo.

Las dosis crecientes de LA aplicadas provocaron alteraciones químicas y químico-físicas en las muestras de suelo que influenciaron en la fitodisponibilidad y disponibilidad del $\mathrm{Cd}$.

La absorción de Cd del lodo albañal por las plantas aumentó con las dosis crecientes de lodo y con la disminución del pH en el suelo.

\section{AGRADECIMIENTOS}

À FAPESP (Fundação de Amparo à Pesquisa do Estado de São Paulo) por el apoyo financiero. 


\section{REFERENCIAS}

1. Malavolta, E.; Fertilizantes e seu impacto ambiental. Micronutrientes e metais pesados, mitos, mistificação e fatos, Produquímica: Piracicaba, 1994.

2. Alloway, B. J., ed.; Heavy Metals in Soils, $2^{\text {nd }}$ ed., Blackie Academic \& Professional: London, 1995.

3. Kabata-Pendias, A.; Pendias, H.; Trace Elements in Soils and Plants, CRC Press: Boca Raton, 1992.

4. Silva, F. C.; Boaretto, A. E.; Berton, R. S.; Zotelli, H. B.; Pexe, C. A.; Bernardes, E. M.; Pesq. Agropec. Bras. 2001, 36, 831.

5. Oliveira, F. C.; Mattiazzo, M. E.; Sci. Agric. 2001, 58, 581.

6. Nascimento, C. W. A.; Barros, D. A. S.; Melo, E. E. C.; Oliveira, A. B.; Rev. Bras. Ciênc. Solo 2004, 28, 385.

7. McBride, M. B.; Barrett, K. A.; Kim, B.; Hale, B.; Soil Sci. 2006, 171, 21.

8. Hooda, P. S.; Alloway, B. J.; Sci. Total Environ. 1994, 149, 39.

9. Smolders, E.; Brans, K.; Földi, A.; Merckx, R.; Soil Sci. Soc. Am. J. 1999, 63,78 .

10. Larsen, S.; Plant Soil 1952, 4, 1.

11. Echevarria, G.; Klein, S.; Fardeau, J. C.; Morel, J. L. ; Comptes Rendus de l'Académie des Sciences, 1997, 324, 221.

12. Hutchinson, J. J.; Young, S. D.; McGrath, S. P.; West, H. M.; Black, C. R.; Baker, A. J. M.; New Phytol. 2000, 146, 453.

13. FAO; World reference base for soil resources, UNESCO: Paris, 1994.

14. Empresa Brasileira de Pesquisa Agropecuária; Sistema brasileiro de classificação de solos, Embrapa Produção de Informação, EMBRAPA Solos: Rio de Janeiro, 1999

15. Empresa Brasileira de Pesquisa Agropecuária; Manual de métodos de análise de solo, $2^{\mathrm{a}}$ ed., EMBRAPA, CNPS: Rio de Janeiro, 1997.

16. Camargo, O. A.; Moniz, A. C.; Jorge, J. A.; Valadares, J. M. A. S. Métodos de análise química, mineralógica e física de solos do Instituto Agronômico de Campinas., IAC: Campinas, 1986 (Boletim Técnico, 106).

17. van Raij, B.; Quaggio, J. A.; Cantarella, H.; Ferreira, M. E.; Lopes, A. S.; Bataglia, O. C.; Análise química do solo para fins de fertilidade, Fundação Cargill: Campinas, 1987.

18. van Raij, B.; Cantarella, H.; Quaggio, J. A.; Furlani, A. M. C. Recomendações de adubação e calagem para o Estado de São Paulo, $2^{\mathrm{a}}$ ed., Instituto Agronômico de Campinas: Campinas, 1996 (Boletim Técnico, 100).

19. Companhia de Tecnologia de Saneamento Ambiental; Relatório de Estabelecimento de Valores Orientadores para Solos e águas Subterrâneas no Estado de São Paulo, CETESB: São Paulo, 2001.
20. Companhia de Tecnologia de Saneamento Ambiental; Decisão de Diretoria $N^{o}$ 195, CETESB: São Paulo, 2005.

21. Boaventura, G. R.; Santos, A. R.; Pinelli, M. P.; Dias, R. P.; Anais do $25^{\circ}$ Congresso Brasileiro de Ciência do Solo, Viçosa, Brasil, 1995

22. Kiehl, E. J.; Fertilizantes orgânicos, Ceres: Piracicaba, 1985

23. Andreoli, C. V.; Sperling, M.; Fernandes, F.; Lodo de esgotos: Tratamento e disposição final. Princípios do tratamento biológico de águas residuárias, UFMG: Belo Horizonte, 2001, vol. 6 .

24. Companhia de Tecnologia de Saneamento Ambiental; Norma P 4.230, CETESB: São Paulo, 1999

25. Lindsay, W. L.; Norvell, W. A.; Soil Sci. Soc. Am. J. 1978, 42, 421.

26. Bataglia, O. C.; Furlani, A. M. C.; Teixeira, J. P. F.; Furlani, P. R.; Gallo, J. R.; Métodos de análise química de plantas, Instituto Agronômico de Campinas: Campinas, 1983

27. Muraoka, T. Em Metodologia de pesquisa em fertilidade do solo; Oliveira, A. J.; Garrido, W. E.; Araujo, J. D. de; Lourenço, S., eds.; EMBRAPA, SEA: Brasília, 1991, cap. 6.

28. SAS Institute; SAS system for Windows. Release 8.02, SAS Institute: Cary, NC., 2001.

29. Havlin, J. L.; Beaton, J. D.; Tisdale, S. L.; Nelson, W. L.; Soil fertility and fertilizers: An introduction to nutrient management, $7^{\text {th }}$ ed., Prentice Hall: New Jersey, 2005.

30. Simonete, M. A.; Kiehl, J. C.; Sci. Agric. 2002, 59, 555.

31. Pierangeli, M. A. P.; Guilherme, L. R. G.; Oliveira, L. R.; Curi, N.; Silva, M. L. N.; Pesq. Agrop. Bras. 2003, 38, 737

32. Fassbender, H. W.; Química de suelos, con énfasis en suelos de América Latina, IICA: Turrialba, 1975.

33. Sanyal, S. K.; DeDatta, S. K.; Adv. Soil Sci. 1991, 16, 1.

34. Novais, R. F.; Smith, T. J.; Fósforo em solo e planta em condições tropicais, UFV, DPS: Viçosa, 1999.

35. Malavolta, E.; Elementos de nutrição mineral de plantas, Agronômica Ceres: São Paulo, 1980

36. Marschner, H.; Mineral nutrition of higher plants, Academia: London, 1995.

37. McLaughlin, M. J.; Singh, B. R.; Developments in plant and soil science Cadmium in soils and plants, Kluger Academic Publishers: Dordrecht, 1999.

38. Berton, R. S. Em Impacto ambiental do uso agrícola do lodo de esgoto; Bettiol, W.; Camargo, O. A., eds.; Embrapa Meio Ambiente: Jaguariúna, 2000, cap. 16 . 